\title{
Os benefícios do aplicativo FisioBerg na prevenção de quedas em Idosos
}

\author{
The benefits of the FisioBerg app in preventing falls in the elderly \\ Los beneficios de la aplicación FisioBerg en la prevención de caídas en ancianos
}

Recebido: 17/08/2021 | Revisado: 22/08/2021 | Aceito: 24/08/2021 | Publicado: 25/08/2021

\author{
Marcos Adiel Morais Castro \\ ORCID: https://orcid.org/0000-0001-6384-6810 \\ Instituto Educacional Santa Catarina, Brasil \\ Faculdade Guaraí, Brasil \\ E-mail: marcosadiel11@gmail.com \\ Lucas Cristh de Sousa Rego Leite \\ ORCID: https://orcid.org/0000-0002-2634-099X \\ Instituto Educacional Santa Catarina, Brasil \\ Faculdade Guaraí, Brasil \\ E-mail: lucascristh@gmail.com \\ Karla Camila Correia da Silva \\ ORCID: https://orcid.org/0000-0003-1538-7028 \\ Instituto Educacional Santa Catarina, Brasil \\ Faculdade Guaraí, Brasil \\ E-mail: karlacamilac@yahoo.com.br \\ Danilo Cavalcante Gonçalves \\ ORCID: https://orcid.org/0000-0002-7744-7842 \\ Faculdade de Palmas, Brasil \\ E-mail: danilocavalcante2006@hotmail.com
}

\begin{abstract}
Resumo
O envelhecimento é um processo biológico inevitável que tem como fundamento alterações estrutural do organismo, sendo um processo progressivo e singular envolvendo mecanismos e a incapacidade de realizar funções. Os casos de quedas se consolidaram um problema na saúde pública, pelo fato de sua alta incidência e consequentes complicações para a saúde e os custos assistenciais que geram. A fisioterapia contribui na prevenção das quedas em idosos, melhorando a funcionalidade, propriocepção e equilíbrio, marcha, força muscular, promovendo o atributo de vida física e mental. De acordo com as informações colhidas, o aplicativo FisioBerg contribui no aumento da dinâmica da avaliação, colhendo informações e no fim possibilitando um possível diagnóstico, assim acelerando todo o processo. O aplicativo é baseado na escala de equilíbrio de Berg e na escala visual analógico, que ajuda os profissionais da área da saúde na avaliação do risco de quedas e dor em idosos. Possui compartilhamento de informações e resultados, apresenta a escala EVA, é versátil, fácil e rápido, possuindo os principais instrumentos para identificar o risco em quedas de idosos, de simples operação, gerando uma intenção positiva a aderência do profissional de saúde e acadêmicos na sua utilização.
\end{abstract}

Palavras-chave: Envelhecimento; Quedas; Fisioterapia; Idosos; Saúde; Prevenção; Aplicativo.

\begin{abstract}
Aging is an inevitable biological process that is based on structural changes in the organism, being a progressive and singular process involving mechanisms and the inability to perform functions. The cases of falls have become a problem in public health, due to the high incidence and consequent complications for health and the care costs they generate. Physiotherapy contributes to the prevention of falls in the elderly, improving functionality, proprioception and balance, gait, muscle strength, promoting physical and mental quality of life. According to the information collected, the FisioBerg application contributes to increasing the dynamics of the assessment, gathering information and ultimately making possible a diagnosis, thus speeding up the entire process. The application is based on the Berg balance scale and the visual analog scale, which helps healthcare professionals in assessing the risk of falls and pain in the elderly. It has information and results sharing, it presents the EVA scale, it is versatile, easy and fast, having the main instruments to identify the risk of falls in the elderly, of simple operation, generating a positive intention the adherence of the health professional and academics in their use.
\end{abstract}

Keywords: Aging; Falls; Physiotherapy; Elderly; Health; Prevention; Application.

\section{Resumen}

El envejecimiento es un proceso biológico inevitable que se basa en cambios estructurales en el organismo, siendo un proceso progresivo y singular que involucra mecanismos e incapacidad para realizar funciones. Los casos de caídas se han convertido en un problema de salud pública, debido a la alta incidencia y las consiguientes complicaciones para la salud y los costos de atención que generan. La fisioterapia contribuye a la prevención de caídas en los ancianos, 
mejorando la funcionalidad, la propiocepción y el equilibrio, la marcha, la fuerza muscular, favoreciendo la calidad de vida física y mental. Según la información recopilada, la aplicación FisioBerg contribuye a incrementar la dinámica de la evaluación, recabando información y en última instancia posibilitando un diagnóstico, agilizando así todo el proceso. La aplicación se basa en la escala de equilibrio de Berg y la escala analógica visual, que ayuda a los profesionales sanitarios a evaluar el riesgo de caídas y dolor en los ancianos. Cuenta con intercambio de información y resultados, presenta la escala EVA, es versátil, fácil y rápido, teniendo los principales instrumentos para identificar el riesgo de caídas en los ancianos, de simple operación, generando una intención positiva la adherencia del profesional de la salud y académicos en su uso.

Palabras clave: Envejecimiento; Caídas; Fisioterapia; Anciano; Salud; Prevención; Solicitud.

\section{Introdução}

Envelhecimento pode ser compreendido como um conjunto de alterações estruturais e funcionais desfavoráveis do organismo que se acumulam de maneira progressiva, especificamente em função do avanço da idade. Essas modificações prejudicam o desempenho de habilidades motoras, dificultando a adaptação do indivíduo ao meio ambiente, desencadeando modificações de ordem psicológica e social (Candeloro, 2007). Nota-se que, por esse motivo vários acidentes domésticos acontecem, como os frequentes casos de quedas. A queda é definida como uma falta de capacidade para corrigir o deslocamento do corpo, durante seu movimento no espaço. As quedas entre pessoas idosas constituem um dos principais problemas clínicos e da saúde pública devido a sua alta incidência, às consequentes complicações para a saúde e aos custos assistenciais (Carregaro \& Toledo, 2008).

O expressivo aumento do número de idosos acarreta impactos e desafios políticos, socioculturais e econômicos para acatar às demandas desse crescente contingente6. Uma delas é a participação de forma mais efetiva na sociedade, com inclusão nas atividades pertinentes à tecnologia (Carregaro \& Toledo, 2008), já que, apesar de haver um aumento da ascensão à internet e do uso de tecnologia pelo idoso a cada ano, eles ainda são o grupo social mais exclusão digitalmente (Gazzola, et al. 2006). Dentre os recursos tecnológicos que despertam o interesse do público idoso, os smartphones têm se destacado por incorporarem, ao celular, funcionalidade de computador, como conexão à internet e possibilidade de uso de aplicativos (Miceli 2002).

A utilização de ferramentas tecnológicas no que abrange a saúde está em crescente expansão (Muellmann, Forberger, Möllers et al. 2016). Uma das principais vantagens das intervenções em saúde por meio de aplicativos é que elas são facilmente acessíveis e utilizáveis, além de alcançarem diferentes segmentos da população (Lima Neto, Goldenberg, Jucá, 2003), como os indivíduos idosos. Para esta parte da sociedade, os aplicativos funcionam como uma estratégia que facilita o autocuidado, da conservação da autonomia e da independência (Miyamoto 2004). Com esse intuito podemos citar alguns aplicativos (APP), como por exemplo: o Alzhe alert, desenvolvido para estimar o perigo de uma pessoa desenvolver a doença do Alzheimer ao longo dos anos, dependendo de seus hábitos e atividades diárias (Jensen, Karroly, Braver. 1986.); e o ADep (Ayuda para depression em espanhol), programa de acesso livre sobre psicoeducação e intervenção cognitivo-comportamental para depressão, produzido no México, como citado pelo Instituto Brasileiro de Geografia e Estatística (IBGE) 2018. No Brasil, outros exemplos desses tipos de aplicativo são o Frágil Mobi, que procura identificar as características do sinal da fragilidade na pessoa idosa, e o aplicativo CuidarTech "Exame dos Pés", ferramenta para auxiliar na avaliação e classificação dos riscos da pessoa com diabetes mellitus desenvolver pé diabético (Newstead et al. 2005).

Percebe-se que, dentre os aplicativos que se encontram disponíveis nas plataformas digitais, encontra-se um aplicativo que foi criado para dispositivo móvel capaz de auxiliar o profissional da saúde na avaliação do risco de quedas em pessoas na terceira fase de vida, possibilitando não só o suporte na avaliação e classificação de risco de quedas, como também permite um gerenciamento mais próximo das fichas dos pacientes avaliados, auxiliando o acréscimo de projetos em saúde que visem à prevenção desses agravos de forma mais veloz e eficaz. Esta ferramenta web mobile denominada FisioBerg tem os dados disponíveis, a qualquer hora e em qualquer lugar, de forma ágil e seus resultados podem ser armazenados no próprio dispositivo ou em um e-mail previamente cadastrado. Este recurso foi desenvolvido atraves de um software tipo aplicativo, em português, 
baseado na escala de equilíbrio de Berg que avalia o risco de queda e dor, facilitando o trabalho de avaliação do risco de queda em idosos por profissionais especializados, acadêmicos e os próprios idosos, reduzindo o impacto na saúde pública.

O APP acima citado utiliza um padrão de programação diferente do convencional, o Open Blocks, baseado em blocos gráficos que representam comandos equivalentes aos utilizados em um ambiente de programação tradicional. Isso é possível pelo meio de uma biblioteca chamada Blocks JAVA que é distribuída pela Scheller Teacher Education Program (STEP) e atrelada a licença do MIT. Os requisitos funcionais definem as funções que o software é capaz de realizar através de inserção de dados, e saída de dados. Tais requisitos definem o comportamento do sistema após o usuário acionar uma função do software. Nota-se que, o sistema realiza o somatório de pontos de acordo com as respostas das 14 questões do formulário preenchido pelo usuário, o resultado desse somatório deve mostrar as chances de queda do indivíduo, alem de possibilitar o compartilhamento de informações; oferece uma opção para indicar o grau de dor do paciente que deve variar de 0 a 10 (Escala EVA); disponibiliza uma edição dos dados é a possível exclusão dos mesmos.

Desta forma, o APP FisioBerg mostra-se uma ferramenta viável para utilização no processo de avaliação, acompanhamento e pesquisa do risco da queda e dor em idosos, com flexibilidade para ampliação do seu desígnio para outras funcionalidades, tais como sincronia com prontuários eletrônicos. Deste modo, o aplicativo demonstra seu fácil acesso e manuseio para os profissionais da saúde, acadêmicos é o público em geral, além de estar disponível, sem custos, para os usuários interessados.

\section{Metodologia}

Este estudo trata-se de uma revisão bibliográfica e descritiva de abordagem qualitativa. Tendo como base para sua discussão teórica, artigos científicos e demais produções intelectuais (Pereira et al., 2018).

Observa-se que, para a realização deste trabalho foi usado como base o APP desenvolvido por um pesquisador fisioterapeuta, com o intuito de descrever de maneira simples e clara o funcionamento deste recurso, trazendo a público de uma forma teórica os programas que o aplicativo FisioBerg utiliza em seu desenvolvedor, fazendo com que fique clara a facilidade para sua utilização.

Obtendo-se tais informações, os estudos mostram as principais qualidades que esses recursos tecnológicos trazem consigo, a rapidez que se obtém no momento da anamnese mostrando um possível diagnostica, facilitando e agilizando este processo.

\section{Resultados e Discussão}

\subsection{Aplicativo FisioBerg}

Os acidentes domésticos se apresentam de várias formas, dentre elas a mais comum são as quedas. Nota-se que, tais acontecimentos podem acontecer em todas as fases da vida, mas, tem um maior índice na terceira idade. Com esse fato em mente o APP FisioBerg, baseado na escala de equilíbrio de Berg e na escala visual analógica, que auxilia o profissional da saúde na avaliação do risco de quedas e dor em idosos, apresentou um estudo de desenvolvimento tecnológico de um aplicativo que foi criado utilizando a ferramenta AppInventor, com as heurísticas de uso para o público em foco (profissionais de fisioterapia e acadêmicos) podendo abranger os demais públicos através da auto avaliação.

Denota-se que, este aplicativo possui compartilhamento de informações e resultados, apresenta a escala EVA, como diferencial além de não apresentar propagandas, fazendo o aplicativo apresentar-se a sociedade em uma forma versátil, rápida, que possui os principais instrumentos para identificar os riscos de quedas em idosos, de fácil operação, gerando uma tendência positiva a aderência do mesmo. 
Figura 1. Tela inicial do APP.

Fisioberg
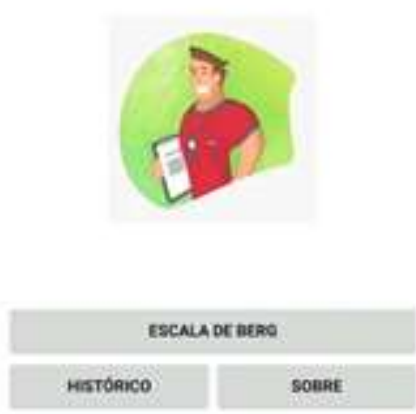

Fonte: https://play.google.com/store/apps/details?id=appinventor.ai_fedegakgb.Fisio.

Observa-se que, o sistema e composto por um QUIS (ferramenta que foi desenvolvida por uma equipe multidisciplinar de pesquisadores do Human-Computer Interaction Laboratory (HCIL) da University of Maryland, com o desígnio de estimar a satisfação subjetiva dos usuários focando aspectos específicos da interface humano-computador) de perguntas, com 14 questões, onde a somatória de pontos utiliza o cálculo de ajuste com a Escala de Berg. O resultado dessa soma deve mostrar as chances de queda do indivíduo; Compartilhamento de informações; Escala visual analógica (EVA), indicando o grau de dor do paciente que deve variar de 0 a 10; edição dos dados, onde se pode atualizar os resultados da Escala de Berg e da escala EVA; e exclusão dos dados, onde possui uma opção para exclusão dos dados inseridos no banco de dados.

Figuras 3 e 4. Perguntas que o APP dispõe.

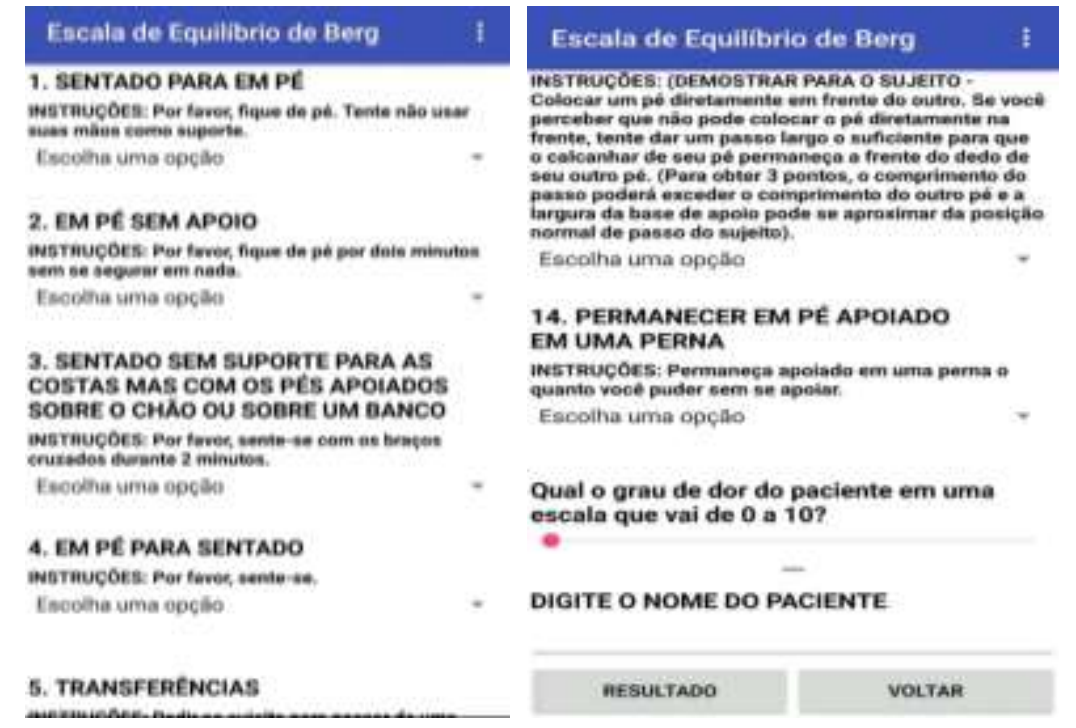

Fonte: https://play.google.com/store/apps/details?id=appinventor.ai_fedegakgb.Fisio 
Apresentando notáveis facilidades e singular desempenho, o APP mostra-se funcional, sendo uma ferramenta viável para utilização no processo de avaliação, acompanhamento e pesquisa do risco de quedas e dor em senis, destacando-se como um auxilio impar na questão de prevenção de possíveis riscos a integridade física.

\subsection{Escala de Berg}

A Escala de Berg é um instrumento validado, de avaliação funcional do equilíbrio composta de 14 tarefas com cinco itens cada e pontuação de 0-4 para cada tarefa: 0 - é incapaz de realizar a tarefa e 4 - realiza a tarefa independente. O escore total varia de 0-56 pontos. Quanto menor for a pontuação, maior é o risco para quedas; quanto maior, melhor o desempenho. (Gazzola et al., 2006, Christofolett et al., 2006).

A escala de equilíbrio de Berg está diretamente relacionada a outros testes de equilíbrio e mobilidade, apresentando uma confiabilidade de teste de 98\% (Berg, Wood-Dauphinnee, Williams, Maki. Measuring. 1992). Outra particularidade dessa escala é a relação não-linear entre a pontuação e o risco de queda correspondente. Os escores variam de 0 a 56 e, quanto maior o escore, melhor o equilíbrio do sujeito avaliado (Berg, Wood-Dauphinnee, Williams, Maki. Measuring. 1992). Assim, cada ponto a menos na escala corresponde a um aumento do risco de quedas; entre os escores 56 a 54, cada ponto a menos é associado a um aumento de 3 a $4 \%$ no risco de quedas; entre 54 e 46, a um aumento de 6 a $8 \%$ de chances, sendo que abaixo de 36 pontos o risco de quedas é de quase $100 \%$. (Newstead, Hinman \& Tomberlin, 2005).

A escala foi adaptada para aplicação no Brasil por Miyamoto et al. (2004), apresentando em cada item escores de 0-4 e um tempo determinado para cada tarefa; tem como pontuação para risco de quedas escore abaixo de 45 pontos.

\subsection{Escala Visual Analógica}

Observa-se que, segundo a International Association for the Study of Pain (IASP), a dor foi oficialmente definida em 1986 como sendo uma "desagradável experiência sensorial e emocional associada a um dano atual ou potencial do tecido ou descrita em termos deste dano" "Miceli (2002)". Noa-se que, no ano de 1996, a American Pain Society (APS) a introduziu como “o $5^{\circ}$ sinal vital” (Galvão, Silva, 2005, Bermudez et al., 2003).

A EVA pode ser classificada como uma mensuração unidimensional, por avaliar somente uma das dimensões da dor, a intensidade (Lima Neto, 2003). A EVA consiste em uma linha horizontal com 10 centímetros de comprimento, assinalada em uma de suas extremidades a classificação "SEM DOR" e, na outra, a classificação "Dor Máxima" (Souza, 2002).

Jensen, Karroly e Braver (1986) falam que, para pessoas jovens, esta escala mostra alto índice de legitimidade e confiabilidade, só que com o acréscimo da idade está relacionado ao aumento de respostas imperfeitas para a EVA.

\section{Conclusão}

A Tecnologia veio para ajudar o homem, e nada mais justo do que a utilizar em prol da saúde da sociedade. Com este pensamento, e com a evolução constante dos meios tecnológicos, principalmente no que se refere à saúde pública, este novo conhecimento favorece e facilita tanto a anamnese, diagnostico, e um possível tratamento. Nota-se que, o aplicativo abortado neste estudo mostra todas as qualidades necessárias para um bom desenvolvimento da entrevista inicial.

Contudo observa-se que, o aplicativo FisioBerg se mostrou como um meio de facilitação que gera à agilidade necessária no momento do primeiro contado com o paciente ou até mesmo em questão da auto avaliação, promovendo um resultado satisfatório que norteia o usuário para o possível diagnostico, indicando possíveis complicações e demostrando recursos que pode ser utilizado para o tratamento do mesmo. 


\section{Agradecimentos}

Agradecemos aos nossos familiares e ao nosso professor e orientador Danilo Cavalcante Gonçalves pelo apoio, ajuda e colaboração para a criação deste estudo que levou a ideia deste artigo.

\section{Referências}

Berg, K. O., Wood-Dauphinnee, S. L., Williams, J. T., \& Maki, B. (1992) Measuring balance in the elderly: validation of an instrument. Can J Public Heath.

Candeloro, J. M. \& Caromano, F.A. (2007) Efeito de um programa de hidroterapia na flexibilidade e na força muscular de idosas. Revista Brasileira de Fisioterapia.

Carregaro, L. R. \& Toledo, A. M.(2008) Efeitos fisiológicos e evidencias cientificas da eficácia da Fisioterapia Aquática. Revista Movimenta.

Carvalho, E., Arantes, R. C., \& Cintra, A. S. R. A inserção de idosos do Instituto Henrique da Silva Semente (IHESS) no município de Indaiatuba/SP na era digital: contribuições fisiogerontológicas. Rev Bras Geriatr Gerontol. 19(4):567-75.

Christofolett, G. (2006) Risco de quedas em idosos com doença de Parkinson e demência de Alzheimer: um estudo transversal. Revista Brasileira de Fisioterapia.

Galvão, O., \& Silva, M. G. (20050 Validade e fidedignidade preliminares da EVA modificada para a população idosa. EssFisionline, Setúbal.

Gazzola, J. M. et al. (2006) Fatores associados ao equilíbrio funcional em idosos com disfunção vestibular crônica. Revista Brasileira de Otorrinolaringologia, São Paulo.

Instituto Brasileiro de Geografia e Estatística (IBGE). Fundo da População das Nações Unidas. (Brasil). Indicadores sociodemográficos: prospectivos para Brasil 1991-2030. https://ww2.ibge.gov.br/home/estatistica/populacao/projecao_da_populacao/publicacao_UNFPA.pdf

Instituto Brasileiro de Geografia e Estatística (IBGE). Pesquisa nacional por amostra de domicílios: síntese de indicadores 2013. http:// biblioteca.ibge.gov.br/visualizacao/livros/liv94414.pdf

Jensen, M. P., Karroly, P.; \& Braver, S. (1986) The measurement of clinical pain intensity: a comparison of six methods. Pain, Amsterdam.

Lara, M. A., Tiburcio M., Aguilar Abrego, A., \& Sánchez-Solís, A. A four-year experience with a Web-based self-help intervention for depressive symptoms in Mexico. Rev Panam Salud Pública. 2014;35(5-6):399-406.

Lima Neto, E. V.; Goldenberg, A., \& Jucá, M. J. (2003) Resultados imediatos da herniorrafia inguinal com anestesia local associada com sedação.

Méndez-Sanz, R., de la Torre-Díez, I., \& López-Coronado, M. (2016) What is your risk of contracting Alzheimer's disease? A telematics tool helps you to predict it. J Med Syst..

Miceli, A. V. P. (2002) Dor crônica e subjetividade. Revista Brasileira de Cancerologia,

Miyamoto, S. T. (2004) Brazilian version of the Berg balance escale. Braz. Med. Biol.

Muellmann, S, Forberger, S, Möllers, T, Zeeb, H, \& Pischle, C. R. Effectiveness of eHealth interventions for the promotion of physical activity in older adults: a systematic review protocol. BioMed Central, 5:47.

Newstead, A. H., Hinman, M. R., \& Tomberlin, J. A. Reliability of the Berg Balance Scale and balance master limits of stability tests for individuals with brain injury. J Neurol Phys Ther, 2005.

Pereira A. S. et al. (2018). Metodologia da pesquisa científica. UFSM

Souza, J. J., \& Sales, M. B. Tecnologias da informação e comunicação, smartphones e usuários idosos: uma revisão integrativa à luz das teorias sociológicas do envelhecimento. R Kairós Gerontol. 19(4):131-54.

Souza CM, Silva AN. Aplicativos para smartphones e sua colaboração na capacidade funcional de idosos. R Saúde Digit Tecnol Educ [Internet]. 1(1):06-19.

Sousa, F. A. E. F. (2002) Dor: o quinto sinal vital. Revista Latino-Americana de Enfermagem.

Tibes, C., Dias, J., \& Zem-Mascarenhas, S. (2014) Aplicativos móveis desenvolvidos para a área da saúde no brasil: revisão integrativa da literatura. Rev Min Enferm.

Vêscovi, S. D. J. B., Primo, C. C., Sant'Anna, H. C., de Oliveira Bringuete, M. E., Rohr, R. V., do Prado, T. N., et al. (2017) Aplicativo móvel para avaliação dos pés de pessoas com diabetes mellitus. 